\section{Heterogeneous Reactions:} Analysis, Examples, and Reactor Design

by L. K. Doraiswamy and M. M. Sharma, John Wiley and Sons, 1984, Volume 1: Gas-Solid and Solid-Solid Reactions, 538 pp., \$66.95; Volume 2 . Fluid-Fluid-Solid Reactions, 374 pp., $\$ 59.95$.

These large volumes represent a major effort to integrate the theory and industrial examples of multiphase reaction engineering, with emphasis on interactions between mass and heat transport and chemical reaction. The result is the most comprehensive account of this subject, a valuable source of important results, and a useful entry into the literature. The authors deserve credit for attempting to unify the subject, but their success is limited by the state of development of reaction engineering. There are far too few experimental results available for in-depth, quantitative analyses of industrial processes; consequently, the connections between the theoretical and experimental results are not very deep or satisfying. This work highlights the need for more detailed industrial data to allow consolidation of the theoretical progress and better guidance in reactor design.

Some of the newer topics engaging reaction engineers are introduced in the book (e.g., supported enzymes and metal complex catalysts), but the authors have not addressed molecular concepts; typical of books in reaction engineering, this one lacks a chemical orientation. The major technological opportunities are offered by new chemical struc- tures, reaction pathways, and catalysts, not by new reactors or manipulation of interactions of transport and reaction processes; perhaps, this book will challenge future authors to integrate chemical concepts into reaction engineering.

The Doraiswamy and Sharma volumes are well produced and fairly priced. They are a commendable advanced treatise, recommended to practicing engineers and as a supplementary reference for graduate courses in reaction engineering.

B. C. GATES

Department of Chemical Engineering University of Delaware Newark, DE 19716
Bimetallic Catalysts: Discoveries, Concepts, and Applications

by John H. Sinfelt, John Wiley \& Sons, 1983. XI + $164 \mathrm{pp}$.

Bimetallic catalysts find wide application, especially in the petroleum industry. They offer the potential of improved selectivity and better activity maintenance as compared to catalysts containing only one metal. John H. Sinfelt's book, the first in a series of Exxon Monographs, gives a detailed and exciting account of research on bimetallic catalysts from the vantage point of one of the leading pioneers in this area. Although most of the work discussed in the book has already appeared in the open scientific literature, the book brings twenty years of research at Exxon's catalysis laboratories into context and makes it easier for the reader to appreciate the development of concepts and ideas concerning bimetallic catalysts.

The book starts with a review of selectivity aspects of bimetallic catalysts comprised of metals from Group VIII and Ib in hydrocarbon reactions such as hydrogenolysis, hydrogenation, dehydrogenation, and isomerization. Then, the question of bimetallic cluster formation in systems containing immiscible metal components is addressed. Results on highly dispersed, supported ruthenium-copper catalysts are compared with ruthenium-copper single crystal studies carried out by G. Ertl's group in Munich. Special emphasis is placed on a description of how physical probes such as EXAFS can successfully be used to obtain structural information on well dispersed, supported ruthenium-copper and osmium-copper clusters. Subsequently, work on platinum-iridium is reviewed bringing Mössbauer spectroscopy into the picture as an additional physical characterization tool for bimetallic cluster catalysts. The final chapter of the book concentrates on the major industrial application of bimetallic catalysts for reforming of petroleum.

This book makes interesting reading not only for the scientist or engineer directly involved in bimetallic catalysis, but serves as an example of the scientific research endeavor in general, demonstrating how new concepts and insights are developed on the basis of carefully posed questions followed by key experiments, ultimately culminating in important industrial applications

JOHANNES SCHWANK Department of Chemical Engineering The University of Michigan Ann Arbor, MI 48109 